\title{
Chronic pain and understanding the interplay between orthopedic and psychological aspects: a case study
}

\begin{abstract}
Chronic pain is the most common cluster of symptoms presented post-trauma. However the multi-faceted nature of this pain, including its psychological sequelae are often undetected in the medico-legal process until late in the proceedings. A review of the salient factors, a decision-making model and case study illustrate the importance of interplay between orthopedic and psychological assessments.
\end{abstract}

Keywords: chronic pain, helplessness, functional overlay, cognitive-behavioral therapy, psychological factors, waddle tests, non-organic factors, vocalization, psychometric testing
Volume 5 Issue 6 - 2017

\author{
Koch,' Cleary A, ${ }^{2}$ Mackinnon J, ${ }^{3}$ Willows J ${ }^{4}$ \\ 'Hugh Koch, Chartered Psychologist and Director, Hugh Koch \\ Associates, Cheltenham, U.K and visiting Professor, Stockholm \\ University, Sweden \\ ${ }^{2}$ Adam Cleary, Clinical Psychologist, Hugh Koch Associates, UK \\ 3john Mackinnon, Consultant Orthopedic Surgeon, Nuffield \\ Health, UK \\ ${ }_{4}^{4}$ Jon Willows, Clinical Psychologist, Hugh Koch Associates, UK
}

Correspondence: Hugh Koch, Chartered Psychologist, Festival House, Jessop Avenue, UK, Tel 01242263 7I5,

Email hugh@hughkochassociates.co.uk

Received: April 21, 2017 | Published: May 16, 2017

\section{Introduction}

The assessment and treatment of chronic pain presents lawyers, experts and claimants with difficulties resulting in variability in the ability to understand fully the interface between physical and psychological factors. ${ }^{1}$ The first port of call for the claimant lawyer is typically a GP, then an orthopedic assessment that considers the physical aspects of pain and will typically suggest one of three possible evidential opinions: evidence of significant organic/ anatomical structural damage where pain is obvious in its organic/ medical origin and is not controversial; evidence of mild/minor organ city where pain is expected but not significant; or lack of evidence of organ city where ongoing pain is unexpected, unexplained and persistent. In all three cases, the orthopedic surgeon will be sensitive to unusual physical signs (Waddel tests) indicative of 'non-organic factors'. These Waddel's signs ${ }^{1,2}$ include: vocalization, axial pressure on the head; simulated versus true spinal rotation; different straight leg raise when lying versus sitting; a light touch on the back perceived as painful; pain diagrams - illustrating pain outside the body, widespread and different types of pain waddle found these correlated with poor physical outcomes. This may be described by the orthopedic expert as an indicative of magnification (conscious or not) and an opinion of 'Psychological Overlay' with a recommended referral to a psychologist for an assessment of possible psychological factors.

The clinical psychologist with experience of chronic pain patients will assess the psychological aspects of the perceived pain bearing in mind two concepts: magnification (unconscious) and exaggeration (conscious). There are also several key psychological aspects of chronic pain. Firstly, interview behaviour, which may take an overt behavioral form (for example, the manner in which the person enters the room and sits or stands), or non-verbal behaviour, such as expression of pain and gesturing. The patient may also express pain verbally. The main thesis of this paper is that both physical and psychological factors need careful assessment medico-legally, at an early stage in the litigation process. Pain is a physical and perceptual process with sensations of pain accompanied by feelings and thoughts about pain. The experience of and response to pain can be influenced by psychological factors (mood, personality, coping style) and social factors (current relationships, pain experiences) a bio-social-economic model. In the medico-legal context, pain, whether it is acute (less than six months duration) or chronic (six months or longer) is identified by various specialists including orthopedic, clinical psychologists, rheumatologists, neurologist, and neurosurgeon. It is essential that front line 'medical' opinion is enhanced by robust psychological opinion. When patients with low back pain of approximately two months' duration (or less) are examined, the physical model of low back pain often does not constitute significantly to the overall prediction of 12 month outcome. It is the model of 'psychological distress' which often explains the complexity of low back pain. ${ }^{3}$

\section{Back pain, for example, is typically caused by:}

i. Mechanical and traumatic causes e.g. degenerative change, fractures or damage to intervertebral discs.

ii. Inflammatory causes e.g. rheumatoid arthritis, neoplastic causes e.g. secondary deposits.

iii. Metabolic bone disease e.g. osteomalacia, osteopororsis.

iv. Referred pain often from retroperitoneal structures e.g. pancreas, kidney or gastric ulcer.

v. Psychogenic causes.

vi. Infection

Mechanical and traumatic causes are most frequently found in post-accident patients. Many people experience pain in the lumbar (and other) regions following road traffic accidents and will complain that they have lumbago, a slipped disc or backache i.e. pain of a spinal origin. These conditions have variable pathology or aetiology and 
variable structural abnormalities seen on $\mathrm{x}$-ray and other imaging techniques, which may correlate very poorly with clinical symptoms. Patients invariably present with a number of strictly physical problems and, even in the well-adjusted patient, there will be a psychological element to their injury and the pattern of disability they have from it. Patients exhibiting little in the way of abnormal illness behaviour can often satisfactorily be treated by solely physical methods, such as physiotherapy, epidural or facet joint injection, or even surgery, as appropriate. Patients exhibiting significant illness behaviour do very badly with simple physical therapy of this type and are more likely to gain help from psychological intervention. Both orthopedic surgeons and psychologists identify abnormal illness behaviour or 'functional overlay' or so called 'inappropriate reactions' by a patient to his/her symptoms. The case study method in the next section illustrates how chronic pain and its psychiatric aspects can present clinically and medico-legally.

\section{Method}

\section{Using a case study approach}

The first author has published several innovative papers illustrating clinical and medico legal issues in civil compensation claim processes. ${ }^{4-6}$ An early case study published in the Personnel and Medical Injuries Law letter in 2004 illustrated a road traffic accident claimant who developed significant chronic pain with both orthopedic, pain medicine and psychological factors. This case went to court where the opposite expert was requested to clarify their general and disagreement with respect to her chronic pain and its management. The key issues in dispute were as follows.

a) Reliability and consistency of her daily activity levels in 1999 and subsequently from witness statements, clinical records and expert reports and, in court, oral evidence.

b) Explanation of variable pain experience and activity levels (organic vs chronic pain vs conscious exaggeration/malingering)

c) Effects of language and cultural issues on help-seeking and therapy uptake.

d) Interaction between pain tolerance and martial relationship conflict.

e) Predicted effects of focused pain management treatment or pain tolerance, activity levels and employability.

f) Differing prognoses with regard to phased return to work.

g) Credibility of claimant and husband.

h) Complementarily of pain medicine and psychological opinions.

The judgment finally given reflected the interplay between orthopedic and psychological factors and her underlying vulnerability, both physical and psychologically. The second case study illustrates a 48 year old man who was in a road traffic accident collision. He was driving and hit from behind at high speed. He experienced a high level of stress and peri-traumatic dissociation at the scene. He attended hospital where whiplash injuries, neck, shoulder and back injuries and several headaches were diagnosed. When assessed initially medicolegally by a General Practitioner, no psychological symptoms were detected. However, six months later when assessed by the first author, two clusters of psychological symptoms were detected: i. Stress, anxiety and low mood including cognitive impairment

ii. Ongoing pain maintained and exacerbated by poor pain coping strategies, both cognitive and behavioral. This resulted in unconscious magnification of his pain experience.

As a consequence of, his ongoing pain experience, he continued to feel unable to work. The overall medico-legal opinion was as follows:

a. He did experience a recognised psychological disorder as a result of the index accident. viz. A pain disorder with both psychological factors (depression) and a general medical condition (DSM IV 307.89). In reaching the above decision on diagnosis, a range of possible opinions have been considered, namely:-

i. Any appropriate Psychological Disorder as laid out in the DSM IV Classification of Mental Disorders.

ii. Absence of Psychological Disorder, but nevertheless experience of discomforting symptoms.

iii. Normal reaction to index event with no significant disruption.

iv. Presence/Absence of GP attendance and treatment

b. He was still suffering from a recognised psychological disorder currently in terms of clinical depression.

c. His residual and ongoing pain experience was, in all probability, exacerbated by psychological factors to a moderate degree. This contributed to unconscious magnification of symptoms.

d. The duration of significant accident-related psychological symptoms was 6 months and ongoing.

e. There was no relevant pre-accident history which impacted on his accident-related problems.

f. The following interventions were recommended:

A course of cognitive behavioral psychotherapy for pain coping problems arising from the accident (10 sessions)

Psychometric testing supported the presence of a high level of generalized anxiety and low mood (Figure 1).

The following graphs display the raw scores and GAD-7 diagnostic ranges

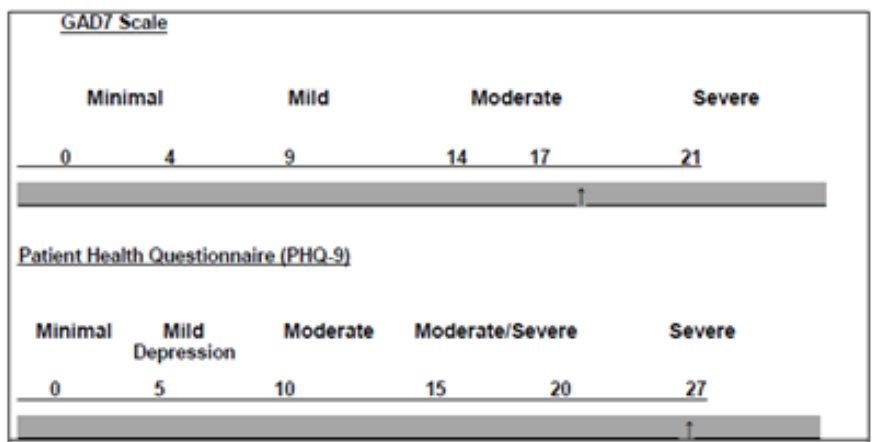

Figure I Psychometric testing.

\section{Psychometric testing}

The following tests, widely used in assessing Post-Accident (Trauma) Stress were administered and Raw Scores and Diagnostic Ranges are shown:- 
I. Scales of GAD-7 Scale and Patient Health Questionnaire (PHQ9) are shown below and relate to his symptoms at the time of the assessment.

II. Clinical Interview Information and back-up information give a consistent and reliable picture of Mr A's response to the accident in April 2013.

$\mathrm{He}$ had endorsed symptoms and psychological problems in a manner which is highly consistent with individuals honestly reporting their difficulties. There was no reason to doubt his veracity in particular about his level of activity. He has shown a degree of consistency in his verbal account. He had insight into his difficulties and attribution was appropriate. There was no evidence of conscious exaggeration.

Additional specific reliability factors were also identified:-

i. Consistency of verbal behaviour.

ii. Consistency of non-verbal behaviour.

iii. Lack of forensic history.

iv. Lack of history of personality disorder.

Mr. A clearly had experienced a distressing and painful accident. Careful, detailed and comprehensive assessment by both psychological and orthopedic experts produced a robust opinion reconciling physical and psychological factors.

\section{Results}

This case study illustrated the importance of clearly defined and valid diagnosis in order to establish the extent of a 'Psychological Head of Damages' for legal purposes. The Chronic Pain Disorder (DSM-IV), more recently replaced by the DSM-V Category Somatic Symptoms Disorder ${ }^{7}$ has the essential features of pain which cause significant distress or impairment in psychological, social and occupational of functioning. Psychological factors play a significant role in the onset, severity, exacerbation or maintenance of pain. This pain is not frequently intentionally produced. Impairment results in inability to work and relationships. Two of three subtypes of pain disorder are:

\section{A. Disorder associated with psychological factors}

B. Disorder associates with both psychological factors and a general medical condition

With a third subtype of disorder associated with a general medical condition (only). Associated mental disorders (e.g. depression, anxiety disorders) may precede the somatic symptom disorder (and possibly predispose the individual to it), occur with it, or result from it. Pain symptoms may be occasionally consciously or intentionally produced. This is uncommon but can occur with overt goals of financial compensation or evasion of criminal prosecutions (DSM $\mathrm{V}$ categories of Factitious Disorder or malingering). Pain can also be experienced in a more general way in terms of a summarization disorder characterised by a combination of pain, gastrointestinal, sexual and pseudo-neurological symptoms with a significant preindex accident history of pain expression. Learned helplessness: a key aspect of chronic pain. A common psychological pain presentation seen in this case study involves how the person 'thinks' about the pain. ${ }^{8}$

A negative or pessimistic way of thinking is often associated with mood disturbance and depression which itself is associated with chronic back pain. This negative way of thinking is often seen within the context of 'Learned helplessness' ${ }^{8,9}$ When a client has experienced acute pain for six months or longer with little or no control of the pain experience, with associated deleterious effects on his/her social and work functioning, he/she develops a style of thinking and behaving which includes:

\section{A. A negative thinking style about self}

B. A negative thinking style about his/her environment including social and working situations

\section{A negative thinking style about his/her future}

D. An avoidance of appropriate behaviour or behaviour which could begin to resolve his/her situation

Pain is frequently and understandably avoided with areas of every life inside and outside the house in order of reduced activity when pain is at its worse.

The typical orthopedic assessment which covers:

\section{i. Previous spinal history}

ii. History of the accident

iii. Evolution of the symptoms

iv. Current symptoms

v. Review of radiographs and other investigations

vi. Diagnosis

vii. And a prognosis is given

The examination includes careful observation of the patient in an informal fashion from meeting them in the waiting room, through to the consulting room and out again, and often observation of them from the clinic car park. Patients present with a number of pain problems which may be significantly accentuated by the psychological aspects of the problems in general. As a general rule, patients with a defined injury with little psychological illness behaviour stand a reasonable chance of doing well and recovering, at least in a physical sense, from their injuries. Patients coping poorly with their physical problem have a poorer prognosis generally to the extent that, even if one is able to resolve their original physical complaint, illness behaviour causes persistence of symptoms and general dysfunction. When conducting a psychological assessment of pain, the expert witness in this case study followed a decision making trail consistent with the adapted 'Road Map' shown below, in Figure 2. ${ }^{9}$

\section{Pain management}

As regards treatment in this case, several psychological interventions have been developed to moderate acute and chronic pain.

These included:

i. Cognitive-behavioral psychotherapy

ii. Biofeedback and relaxation training

iii. Couples Therapy

iv. Counselling and psychotherapy 
Road Map of Psychological Diagnosis of 'Pain'

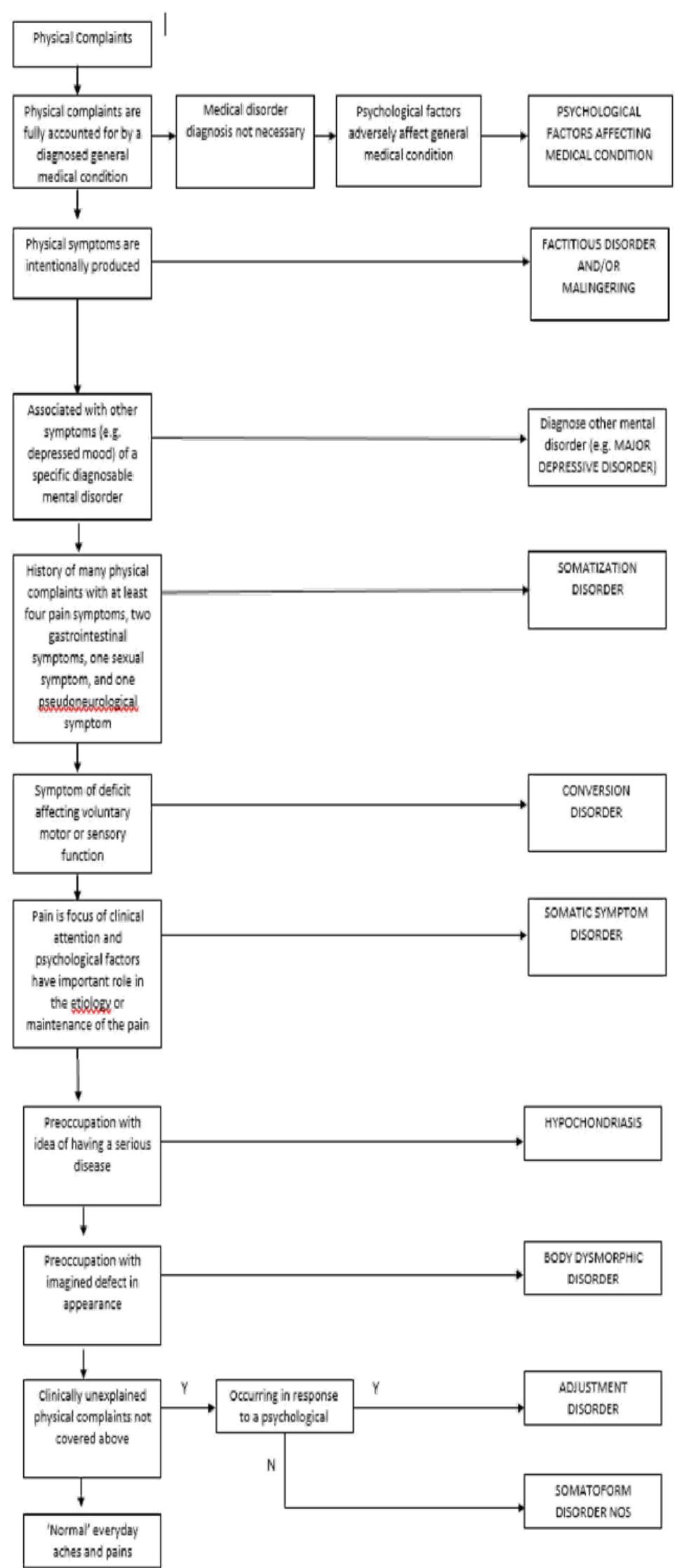

Figure 2 Road map of psychological diagnosis of pain.

Citation: Koch, Cleary A, Mackinnon, et al. Chronic pain and understanding the interplay between orthopedic and psychological aspects: a case study. MOJ Public Health. 20I7;5(6):2I5-22I. DOI: I0.15406/mojph.20I7.05.00I50 
These are complimented by the use of pain relief medication and some anti-depressants which have been empirically found to affect pain experience e.g. amitriptyline.

Multi-disciplinary pain programmes vary widely in their existence and availability in the UK as in the USA. They also vary as do their unidisciplinary counterparts (e.g. cognitive-behavioral therapy) in their empirical effectiveness. It is incumbent on the optimistic claimant, lawyer and expert (both orthopedic and psychologist) to encourage intervention that will in all possibility, let alone probability, lead to partial positive control of pain experience. Untangling Complex Presentations: Physical Conditions Which 'Mimic' Psychological Symptoms. The accurate and reliable assessment of the breadth, severity of pain and duration of psychological symptoms is central to psychological medico-legal enquiry. Within this context, the attribution of psychological symptoms in relation to a given 'index event' (for example a road traffic accident or an injury at work) is of central importance. The practice of medico-legal assessment typically involves an 'untangling' of multiple and complex trauma-related phenomena which frequently have both physical and psychological components. Chronic physical pain can undermine self-esteem, lower mood and also lead to feelings of lethargy and frustration. Phobic anxiety, for example in relation to driving following a severe road traffic accident, is typically experienced in a very physical (or 'somatic') way with an elevated heart rate, surges of adrenalin and hyperventilation. There is, of course, a profound and ever-present connection between mind and body and much room for overlap and misinterpretation when we talk of 'feeling anxious' or 'depressed. ${ }^{10}$

How people talk and think about their chronic low back pain Coping issues for chronic low back pain centre on the finding that claimants tend to strongly believe that their pain has a definable and curable physical cause rather than considering any psychosomatic reasons for their pain. Sufferers of chronic low back pain are often not believed and are seen as malingerers and therefore these people tend to focus on the physical, as the psychological aspects are seen as stigmatizing. However, sufferers experience high incidences of psychological and social difficulties, such as loss of social roles and loss of independence, although they focused much less on these effects.

This paper has implications for the examination of the physical and psychological symptoms of chronic low back pain following an RTA. It is likely that people who present with low back pain will emphasize the physical nature of their symptoms, despite also experiencing psychological and social problems. Psychological examinations are beneficial in order to gain a true picture of their problems. Furthermore, these people may not respond to medical treatment alone, but will require psychological treatment that is sympathetic to their beliefs about the cause of their pain.

\section{Discussion}

This case study illustrates the importance of comprehensive psychological pain assessment, assessment of credibility and truthfulness and the effect of chronic pain on employment as follows:

\section{A. Psychological pain assessment}

The psychological or psychiatrist with a special interest in chronic pain then carries out a full assessment to try to understand what, if any, psychological factors might be contributing to ongoing pain perception, pain intolerance and reduced functioning.

\section{This examination will look for:}

a) Evidence of non-pain related psychological disorder e.g. depression, anxiety, stress and associated stress in the claimant's current life. These may be index accident related or non-index accident related. As we know, the experience of a pain is made worse by other levels of distress. A 'vicious circle' can be set up between pain and stress.

b) Evidence of pain-related psychological disorder in which the claimants thinking, behaviour and communication are partly/all disrupted by their pain experience. These disrupted psycho-social factors then help to maintain pain intolerance, experience and perception which typically results in ongoing disability and pain-avoidance behaviour. Examples of this might be 'the pain is constant and could not be worse' (thinking), 'I cannot do anything and don't do anything at home' (behaviour) and 'you have to help me do everything' (communication).

Both 1) and 2) above will typically result in the 'functional overlay' or 'inappropriate signs' picked up by the orthopaedic expert. Both are legitimate psychological conditions and have practical and/or treatment implications (e.g. CBT within the context of pain management approach). ${ }^{11}$

\section{B. Reliability and truthfulness}

The psychologist and the orthopedic one also look for evidence that the claimants may be consciously exaggerating for some other sort of gain e.g. financial. Both experts and the court have a responsibility to assess the reliability of evidence. Some of the indications of them are:

a. Inconsistency of data (verbal information on one day/verbal information on another; difference between verbal and written self-report; discrepancy of self-report with GP or hospital records).

b. Inconsistency between data and video surveillance report.

Care needs to be taken not to over-emphasise the above 'exaggeration' as intentional, especially in item 1 above other factors such as memory and interview factors need to be taken into account. Having diagnosed a Somatic Symptom Disorder in which both medical and psychological factors play a part, careful history taking can clarify if either factor pre-existed the index accident i.e. previous pain, previous high frequency GP attendance for one or more multi site pain over several years. References in the GP notes to current or past 'abnormal illness' behaviour and diagnosis of a mixed pain disorder or summarization disorder. Review of GP notes is essential. ${ }^{12}$

\section{Chronic pain and employment}

A crucial component of orthopedic and psychological evidence is the prognosis as it relates to occupational ability and 'return to work' (RTW). On a clear day, both Orthopaedic and Psychological experts can state with a level of confidence what, from either an orthopedic or psychological point of view (respectively), the likely future incapacity to work, of what a reasonable duration off work was. However, it is not atypical for there to be a discrepancy between orthopedic or psychological predictions and actual level of perceived pain and occupational activity.

\section{Innovative 'next steps' in medico-legal process}

\section{A. The joint interview: back to back}

There are significant advantages of consecutive orthopedic and psychological interviews on the same day in terms not only of 
claimant convenience (travel, stress) but also term of the opportunity for both clinicians to share clinical diagnostic and prognostic views with each other immediately. This results in a more immediate and reliable sharing of views than would normally occur 6-24months later on sharing of reports which have been complied at different times in the course of a claimant's pain symptoms. The first and third authors have experience of this joint working in Cheltenham and London. ${ }^{13}$

\section{B. The joint opinion: explaining ongoing pain experience}

The majority of 'joint opinions' or 'Heads of Agreement and Disagreement' are compiled by two experts of the same or similar discipline. However, there is an increasing frequency of commissioning cross-specialty joint opinions, typically between psychological/ psychiatric experts and other experts e.g. orthopedic or pain medicine (relating to pain) or neurological (relating to head injury, concussion and cognitive effects). The orthopedic/psychological joint opinion has particular relevance in the Court's quest to explain a claimant's ongoing pain experience. It allows the often-present ambiguity surrounding 'abnormal illness behaviour' and 'behavioral motivation' to be clarified by the experts together, rather than separately via a proliferation of addendum letters or by the lawyers or barristers with informed guess-work. ${ }^{14}$

\section{Robust opinions about chronic pain need robust reasoning}

A series of postulates or standards developed by the first author for conducting medico-legal psychological assessments ${ }^{15}$ sets the scene for obtaining a robust psychological opinion and are shown below in Figure 3. These have been extended below in Figure 4 to include medico-legal postulates relevant to this crucial issue of chronic pain.

I. A robust opinion should address diagnosis, causation and attribution, duration

1. and prognosis.

II. A robust opinion will, whenever possible, include more than one type of evidence. An opinion based on claimant self report only may still be valid but is not considered as robust as an opinion derived from utilising several sources of data ( Len., self report, medical records, impact upon other areas of functioning) in medico legal terms.

III. The classification/diagnostic categories given in DSM 5 and ICD 10 are a part of an expert's opinion /formulation - this systematic check of relevant criteria should be balanced by wider clinical judgment and contemporaneous records, if available.

IV. The expert's Mental State Examination should be consistent with the claimant's description of currently active symptoms - a clear discrepancy reduces the robustness / strength of an opinion.

V. Wherever possible, GP computerized attendance records should be made available to the expert. The subsequent analysis (i.e., evidence of or lack of corroborative data) will increase the strength or reliability of the opinion given.

Vl. A therapist who has alreadv treated a claimant cannot provide an impartial or independent expert opinion on issues of diagnosis, causation or

VII. A robust opinion should include a history of factors which could, on the balance of probabilities, affect a specific index event reaction.

VIII. A robust opinion should give particular emphasis to the 12 month period prior to and post the index event, but not to the exclusion of earlier or later history.

IX. In any interview where the claimant displays a high level of affect, a differential opinion should be made between understandable presentation at interview when recalling a distressing or frustrating event involving perceived injustice and dinically significant adjustment problems which might require intervention,

X. An expert opinion should incrementally increase in robustness over time with access to more data and discussion with other relevant professionals, both legal and clinical.

XI. An expert's opinion should be the 'best fit' professional view of all available data at that time, and should be modified, if appropriate, as and when new data becomes available.

XII. When kev evidence (e.g medical records) is unavailable, the expert should state that the robustness of his/her opinion is reduced as a result.

XIII. It is encumbent on the expert to be impartial, independent of instructing party, and maintain as high level of logicality as possible when appraising evidence.

XIV. Experts should maintain a high level of accessibility to lawyers in order to encourage and not hinder rapid process and resolution of litigation.

XV. Experts should understand and be sympathetic to the claimant's experience of litigation stress, irrespective of their expert opinion on the specificcase.

Figure 3 Medico-legal postulates. 


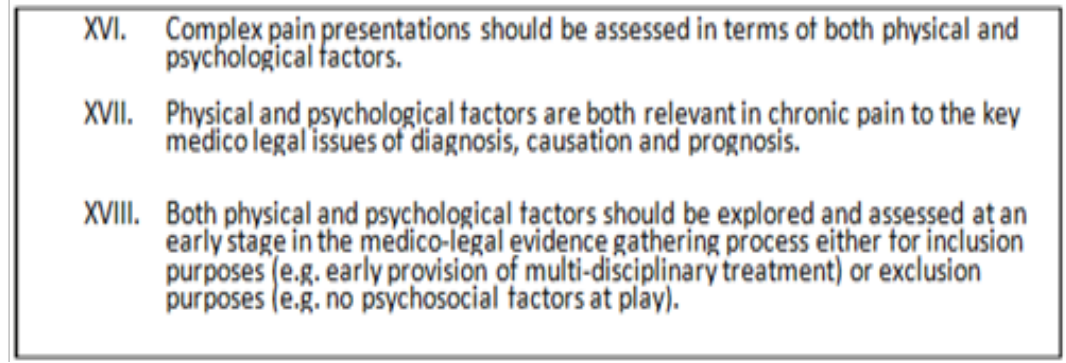

Figure 4 Chronic pain postulates.

\section{Conclusion}

Pain experience and expression are influenced by a number of interacting factors, physical and psychological as illustrated by the case study presented and discussed here. From an orthopedic standpoint, the patient can often have a specific diagnosis of a definable, and perhaps curable, lesion applied, and recommendations to the GP to have the patient followed up in a local orthopedic clinic, can, of course, be very helpful. In many cases, the orthopedic assessment of the patient suggests that there may be little in the way of an organic lesion that is in any way definable and much in the way of psychological problem to be dealt with, and again a formal psychological assessment and treatment of that element of the patient's global problem may assist him significantly. Experts have to be empathetic in their understanding and assessment of clients with chronic pain. Lawyers have to invite and expect comprehensive orthopedic and psychological opinions which assess reliability and validity whether a head of damages of pain disorder/somatic symptom disorder (with psychological factors) exists and whether the client with help can mitigate the associated loss of amenity and earnings by obtaining effective treatment of pain disordered behaviour and experience. Lawyers, Orthopaedic and Psychological/psychiatric experts need to work together to clarify the several types of relevant evidence to reliably understand what pain any one claimant is experiencing. Pain whether, mediated by organic or non-organic factors, typically can be managed better with the appropriate understanding, advice and treatment in the motivated claimant who provides truthful and credible evidence to the court. ${ }^{16}$

\section{Acknowledgements}

None.

\section{Conflict of interest}

Author declares that there is no conflict of interest.

\section{References}

1. Koch HCH, Mackinnon JMM. Taking pain seriously. 1997;7(1):16-18.
2. Waddell G, McCulloch JA, Kummel E, et al. Non-organic physical signs of low back pain. Spine $S .1980 ; 5(2): 117-25$.

3. Rose MJ, Reilly JP, Slade PD, et al. A comparative analysis of psychological and physical models of low back pain experience. Physiotherapy. 1995;81(12):710-716.

4. Koch HCH, Court K, Bates S. Medico-Legal Implications of Assessing Unreliability in Civil Compensation Cases: A Case Study Reflecting Potential Unreliability. Scholarena SAJ Case Reports. 2017.

5. Koch HCH. Medico Legal Case Commentary: Interface between Clinical Opinion and Legal Case Reporting in Personal Injury Litigation. Mathews Open Access Journal. 2016

6. McAllister MJ. Understanding Chronic Pain. Institute for Chronic Pain. Minneapolis, USA; 2017

7. APA. DSM V: Diagnostic and Statistical Manual of Mental Disorders. Washington, USA; 2013.

8. Seligman MEP. Psychology of Pain. London: Wiley; 1997.

9. First MB, Frances A, Pinas HA. DSM-IV Handbook of Differential Diagnosis. APA Washington DC; 1995.

10. Willows J, Williams J. Untangling complex presentation. PIBULJ. 2015.

11. Koch HCH, Mackinnon JM. Understanding ongoing pain. Legal \& Medical. 2007.

12. Koch HCH, Lillie FJ, Kevan T. Perfect Attendance: Decision Making Model for assessing the significance of GP attendance records-LegalMedical. 2006:16-17.

13. Koch HCH. Getting to grips with Chronic Pain: Review of selective, excellent clinical and medico-legal publications. BJN Healthcare Journal. 2016.

14. Koch HCH, Sanders M, Boyd T, et al. Joint Opinion preparation in chronic pain cases. Expert Witness Journal. 2015.

15. Koch HCH. Legal Mind: Contemporary Issues in Psychological Injury and Law. Manchester: Expert Witness Publications; 2016.

16. Koch $\mathrm{HCH}$, Akehurst L, Easton S. Judging Credibility of a Road Traffic Accident Claimant. Journal of Case Reports and Studies. Annex Publishers; 2017. 